\title{
Super iron-rich gas towards GRB 080310: SN yields or dust destruction?
}

\author{
A. De Cia ${ }^{1}$, C. Ledoux ${ }^{2}$, P. Vreeswijk ${ }^{1}$, A. Fox ${ }^{2,3}$, A. Smette ${ }^{2}$, P. \\ Petitjean $^{4}$, G. Björnsson ${ }^{1}$, J. Fynbo ${ }^{5}$, J. Hjorth ${ }^{5}$ and P. Jakobsson ${ }^{1}$ \\ ${ }^{1}$ Centre for Astrophysics and Cosmology, Reykjavík, Iceland - email: annalisa@raunvis.hi.is \\ ${ }^{2}$ European Southern Observatory, Santiago, Chile - ${ }^{3}$ STScI, Baltimore, MD 21218, USA \\ ${ }^{4}$ Institut d'Astrophysique de Paris, France - ${ }^{5}$ Dark Cosmology Centre, Copenhagen, Denmark
}

\begin{abstract}
We performed Rapid-Response Mode (RRM) VLT/UVES high-resolution UV/ optical spectroscopy of the GRB 080310 afterglow, starting 13 min after the burst trigger, in order to investigate the ISM in the GRB host galaxy. The four spectra show remarkable features at $z_{G R B}$, including a low $\log N(\mathrm{HI})=18.7$ and time-variable absorption from ground-state and excited levels of Fe II and Fe III, the latter being observed for the first time in a GRB afterglow. These observations indicate i) ongoing photo-ionization of the surrounding gas due to the GRB radiation and ii) $\mathrm{Fe}$ and $\mathrm{Cr}$ overabundances in the host galaxy ISM. We derive ionic column densities through a four-component Voigt-profile fit of the absorption lines and investigate the pre-burst ionization level of the gas with CLOUDY photo-ionization modelling. The resulting intrinsic $[\mathrm{Si} / \mathrm{Fe}]=-1.4([\mathrm{C} / \mathrm{H}]=-1.3,[\mathrm{O} / \mathrm{H}]<-0.8,[\mathrm{Si} / \mathrm{H}]=-1.2,[\mathrm{Cr} / \mathrm{H}]=+0.7$ and $[\mathrm{Fe} / \mathrm{H}]=$ $+0.2)$ for the whole line profile - and even more extreme for one of the absorption components cannot be explained with current models of SN yields. Dust destruction may contribute to the marked iron overabundance, possibly induced by the burst. The overall high iron enhancement along the line-of-sight also suggests little recent star formation in the host galaxy.
\end{abstract}

Keywords. gamma rays: bursts, galaxies: ISM, galaxies: abundances

\section{Introduction}

Absorption-line spectroscopy of GRB afterglows can probe the gaseous and metal content of the host galaxy ISM out to high redshift (e.g., Prochaska et al., 2007). In particular, high-resolution spectroscopy provides reliable estimates of the metallicity, relative abundances, ionization states and kinematics of the gas along the line-of-sight in the host galaxy (e.g., Ledoux et al., 2009). Moreover, absorption-line variability is witness to the influence of the afterglow radiation on the surrounding medium - bearing information on the distance between the absorbing gas and the GRB - (e.g., Vreeswijk et al., 2007), but this typically requires a prompt response to be observed. RRM VLT/UVES observations of GRB afterglows therefore open unique insights on the properties of GRB-selected galaxies at $z=2-4$, but the current sample is still limited to a handful of cases. Here we summarize remarkable findings from RRM VLT/UVES spectra of the GRB 080310 afterglow. A detailed study of the spectra is presented in De Cia et al. (2012), while photo-excitation/ionization modelling of absorption-line variability due to the GRB is performed in Vreeswijk et al., in preparation.

\section{Overview of the system}

The consecutive four early UVES spectra of GRB 080310 show numerous absorption lines at the GRB host galaxy redshift $(z=2.427)$, including Ly $\alpha$, typical resonance metal lines, Fe II fine-structure lines and a Fe III triplet of lines(UV34) arising from a 
highly-excited level, which has never been observed before in a GRB afterglow. Remarkably, Fe II and FeIII transitions from all (ground-state and excited) levels and the Cr II ground state show time variability. This is a clear indication of ongoing photo-ionization induced by the GRB radiation.

We estimated the ionic column densities with a four-component Voigt-profile fit of the absorption lines and measured a $\operatorname{low} \log N(\mathrm{HI})=18.7$ and a marked overabundance of Fe and $\mathrm{Cr}([\mathrm{Si} \mathrm{II} / \mathrm{Fe} \mathrm{II}]=-1.47 \pm 0.14,[\mathrm{C} \mathrm{II} / \mathrm{Fe} \mathrm{II}]=-1.74 \pm 0.17$ and $[\mathrm{Si} \mathrm{II} / \mathrm{Cr} \mathrm{II}]=$ $-2.03 \pm 0.19$ in one of the absorption components labelled "b"). The pre-burst relative abundances, taking out the effect of ionization due to the GRB and the ambient radiation field, are even lower. We performed CLOUDY photo-ionization modelling of the expected pre-burst ionic column densities (derived in Vreeswijk et al., in prep.) to estimate an intrinsic $[\mathrm{Si} / \mathrm{Fe}]=-1.4$ for the whole line profile, and even more extreme ratios for component "b" ([Si/Fe $] \leqslant[\mathrm{Si}$ II $/ \mathrm{Fe}$ II $]$ and $[\mathrm{C} / \mathrm{Fe}] \leqslant[\mathrm{C}$ II $/ \mathrm{Fe}$ II $]$ ).

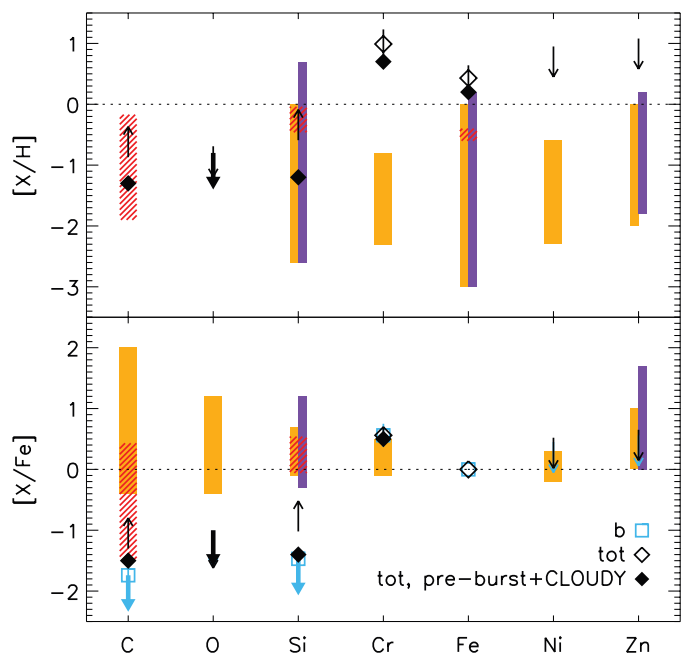
Figure 1 shows the abundances relative to $\mathrm{H}$ (top) and Fe (bottom) estimated for the GRB 080310 absorber (diamonds, squares and arrows). The thick arrows and filled symbols indicate ionization-corrected values, while thin arrows and open symbols refer to the observed ionic abundances. The typical abundances observed in QSO-damped Ly $\alpha$ absorbers (DLAs), GRB DLAs and Lyman limit systems are shown, as comparison, by the rectangular purple, orange and dashed-red regions, respectively. The GRB 080310 absorber clearly shows peculiar abundances produced by $\mathrm{Fe}$ and $\mathrm{Cr}$ overabundances, even more marked in component "b".

\section{Implications}

One possible explanation for the peculiar abundances in the GRB 080310 absorber is that specific Supernovae (SNe) have enriched the ISM with metals in the history of the GRB host galaxy. SN Ia yields can produce [Si/Fe] as low as -0.5 (e.g., Tanaka et al 2011), while [Si/Fe] down to -2.5 are expected for PopIII star SNe, but with strong $\mathrm{Cr}$ depletion (Ohkubo et al. 2006). Thus, current models of SN yields cannot reproduce the observed abundances. An intriguing possibility is that destruction of drifted dust grains may have recycled a significant amount of refractory elements such as Fe and Cr into the ISM. This may be due to the GRB or to other processes such as SN shock waves in star forming regions. In any case, the extreme iron overabundance suggests i) a low amount of dust at the time of observing and ii) little recent star formation along the line-of-sight.

\section{References}

De Cia, A., Ledoux, C., Vreeswijk, P. M., Fox, A. J., Smette, A., et al. 2012, A\&AA, accepted Ledoux, C., Vreeswijk, P. M., Smette, A., Fox, A. J., Petitjean, P., et al. 2009, A\&SA, 506, 661 Ohkubo, T., Umeda, H., Maeda, K., Nomoto, K., Suzuki, T., et al. 2006, ApJ, 645, 1352 Prochaska, J. X., Chen, H., Dessauges-Zavadsky, M., \& Bloom, J. S. 2007, ApJ, 666, 267 Tanaka, M., Mazzali, P., Stanishev, V., Maurer, I., Kerzendorf, et al. 2011, MNRAS, 410, 1725 Vreeswijk, P. M., Ledoux, C., Smette, A., Ellison, S., Jaunsen, A., et al. 2007, A $\mathscr{\mho} A$, 468, 83 